\title{
RELACIONES BIOMETRICAS Y COMPOSICION QULMICA DE ALMEJAS DE AGUA DULCE (Anodontites trapesialis)
}

M. Medina $\mathrm{V} *$

0 . M endieta T. *

\section{RESUMEN}

En el presente trabajo se determinaron al gunas relaciones biométricas para A. trapesialis. Las relaciones biométricas consideradas fueron: (a) longitud total de las valvas versus peso total; (h) longitud total de las valvas versus peso parte comestible; (c) peso total versus peso parte comestible. En todos los casos el ajuste de los datos experimentales se efectuó mediante mínimos cuadrados, empleando el programa Statgraf 4.0.

L as ecuaciones obtenidas para las diferentes relaciones biométricas fueron:

\begin{tabular}{lll}
\hline & Ecuación & Coef. Corr. \\
\hline (a) $W_{T}=$ & $0.13172 * 12,6153$ & 0.873638 \\
(b) $W_{p c}=$ & $1,05439 \operatorname{EXP}(0.276179 * \mathrm{~L})$ & 0.817877 \\
(c) $W_{p c}=$ & $-2.47421+0.359364 * W_{T}$ & 0.811715 \\
\hline
\end{tabular}

Donde:

$\begin{array}{lll}W T & = & \text { Peso total } \\ \mathrm{L} & = & \text { Longitud de las valvas } \\ W_{P C} & = & \text { Peso parte comestible }\end{array}$

La composición química de la parte comestible fue la siguiente: Agua, $84.20 \%$, proteína, $12.60 \%$, grasa, $0.90 \%$, cenizas, $1.90 \%$ y carbohidratos, $0.40 \%$.

Palabras Claves: Biometría, composición química, almejas de agua dulce, A. trapesialis.

* U niversidad Nacional de San M artín-Tarapoto. Facultad de Ingeniería A groindustrial. Casilla Postal 239-Tarapoto 


\section{INTRODUCCION}

La almeja de agua dulce A nodontites trapesialis es un recurso cuyo hábitat natural lo constituyen los cuerpos de agua de la Región San Martín, y cuya población se viene incrementando debido al aumento del área dedicada a la acuicultura, siendo por ello necesario conocer las características biométricas y composición química de las mismas, datos que pueden servir de base para su posterior procesamiento.

A. trapesialis está distribuida extensamente en todo el continente americano, desde Argentina hasta M éxico (2). En el Perú, no existen muchos trabajos sobre esta especie, a pesar que se encuentra en la mayoría de cuerpos de agua de la selva peruana, región en la que son conocidas comúnmente como "cucharas". En la figura 1 se presenta un ejemplar adulto de A. trapesialis.

Con miras al procesamiento de esta especie, el objetivo del presente trabajo fue determinar diferentes relaciones biométricas y la composición química de la parte comestible de la misma.

\section{MATERIALES Y METODOS}

Las almejas utilizadas en el presente trabajo fueron recolectadas en piscigranjas del distrito de Morales, próximo a la ciudad de Tarapoto. El trabajo se efectuó íntegramente en los laboratorios de la U niversidad Nacional de San M artín.

Los pesos se registraron en una balanza de triple barra Ohaus, con aproximación de $0.1 \mathrm{~g}$. Las medidas fueron efectuadas con un malacómetro. Los diferentes valores experimentales de peso y longitud fueron ajustados mediante mínimos cuadrados, empleando el programa estadístico Statgraf 4.0

L a precocción fue realizada en agua hirviente por un lapso de 6 minutos.

$L$ a determinación del contenido de humedad, proteína, grasa y cenizas fue realizado según los métodos aprobados por la (A.O.A.C., 1970)

\section{RESULTADOS Y DISC USION}

\section{RELACIONES BIOMETRICAS}

$L$ a relación longitud de la valva versus peso total se muestra en la Figura 2 , donde $W_{T}$ es el peso total y $L$ es la longitud de las valvas. Para la longitud promedio de $10.6 \mathrm{~cm}$, el peso promedio correspondiente es de $63.26 \mathrm{~g}$. (Campos, 1993) trabajando con especimenes extraídos del Lago Sauce (San Martín), 
reportó para esta relación una curva similar pero, no informa sobre la función matemática que representa a dicha curva.

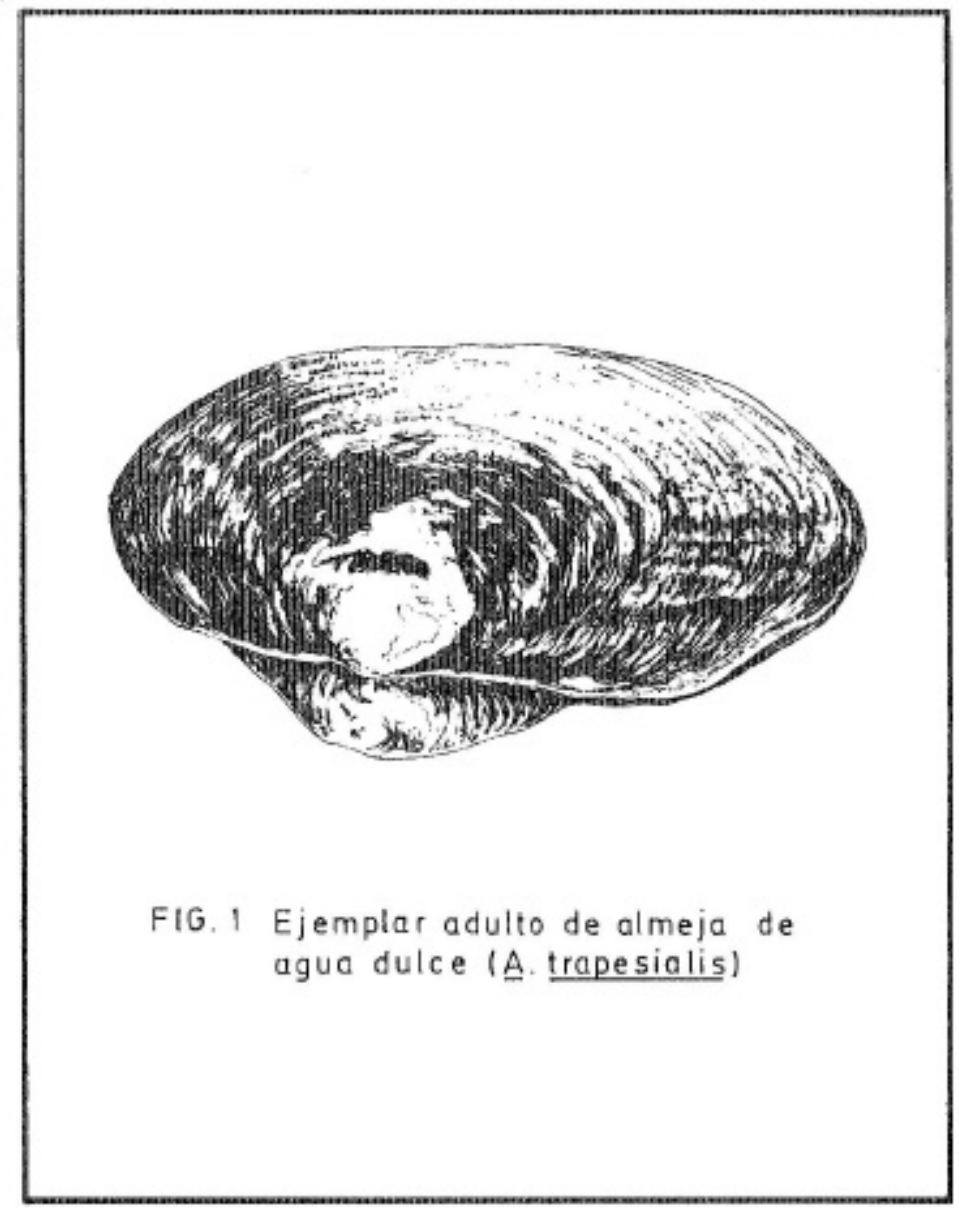




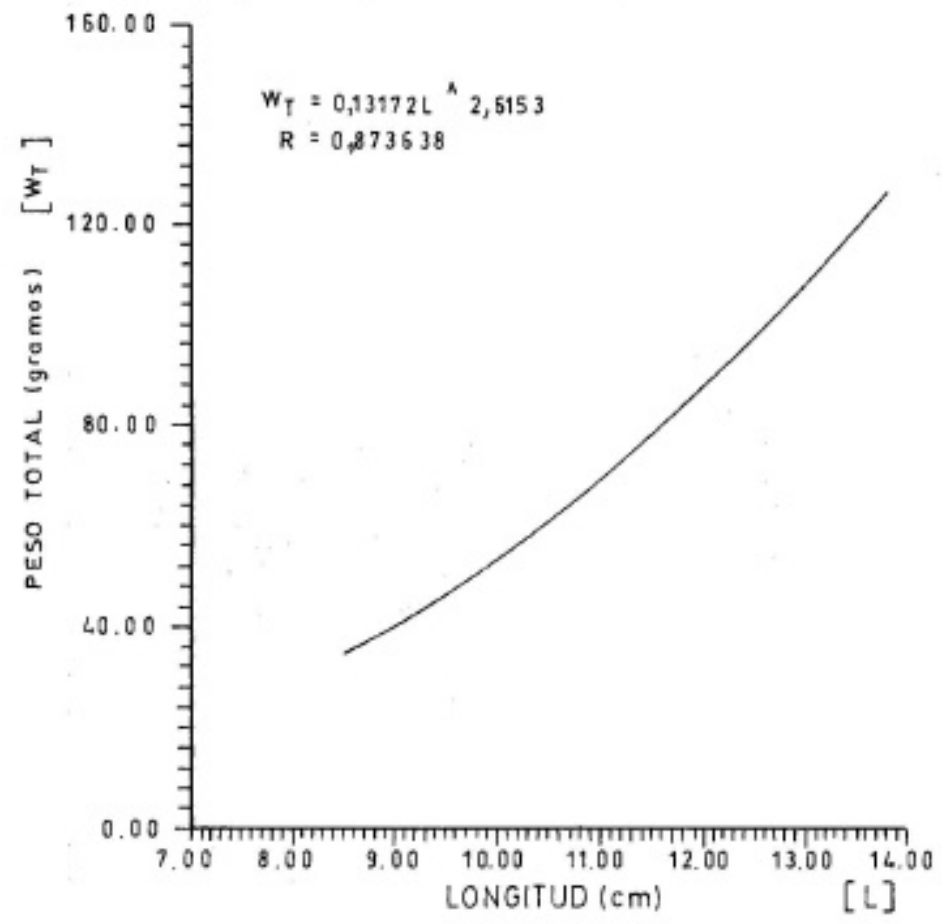

FIG.2 Relación peso total vs longitud de almejas de agua dulce (Anodontites trapesialis) 
La Figura 3 se refiere a la longitud de la valva en relación al peso de la parte comestible, donde W $\mathrm{Pc}$ es el peso de la parte comestible y $L$ es la longitud de las valvas. Para la longitud promedio de $10.6 \mathrm{~cm}$. el peso promedio de la parte comestible es de $19.70 \mathrm{~g}$.

La Figura 4 muestra la relación peso total versus peso de la parte comestible, donde $W_{\mathrm{T}}$ es el peso total y $\mathrm{W}_{\mathrm{p}}$ es el peso de la parte comestible. EI rendimiento de la parte comestible es el $32 \%$ del peso total del individuo, de manera que de $1007 \mathrm{~kg}$ de "cucharas" se obtendrá aproximadamente $32 \mathrm{~kg}$ de parte comestible.

En la Figura 5 se presenta la relación peso total versas peso parte comestible precocida, donde $W_{T}$ es el peso total y $W_{p}$ es el peso de la parte comestible precocida resulta ser igual al $18 \%$ del peso total. Esta relación fue determinada considerando que en la mayoría de formas de procesamiento de bivalvos, se efectúa una precocción para poder extraer la parte comestible con facilidad.

\section{VARIACION DE TALLAS}

La Figura 6 reúne las longitudes de 301 ejemplares colectados, que varían entre 5 y $14 \mathrm{~cm}$ (promedio, $10.6 \mathrm{~cm}$ ). El 76.08\% de la población fue mayor de 9 $\mathrm{cm}$. y considerados adecuados para ser utilizados como materia prima en diferentes procesos. Este resultado difiere ligeramente del reportado por Campos, (1993), para muestras recolectadas en el lago Sauce; pudiendo deberse estas diferencias a factores bioecológicos propios del medio en donde fueron realizados los muestreos.

\section{COMPOSICION QUIMICA}

La composición química proximal de la parte comestible de almejas de agua dulce se presenta en el Cuadro 1. L os valores porcentajes obtenidos para los diferentes componentes son concordantes con los presentados por Campos, (1993) para la misma especie colectada en el lago Sauce. Asimismo, son próximos a los reportados por Collazos, et al (1986), Córdova, (1985), Llanos, (1978) y Sánchez y Lam (1970) para conchas de abanico, almeja blanca, machas y choros crudos, respectivamente. La composición química proximal obtenida indica que se trata de un alimento de buen valor nutritivo, que podría ayudar a mejorar el nivel proteico deficitario de la dieta del poblador sanmartinense, en especial en el sector rural. 


\section{Cuadro 1.}

\section{Composición química de almejas de agua dulce (Anodontites trapesialis)}

Componente

A gua

Proteína

Grasa

Cenizas

Carbohidratos (diferencia)
Porcentaje

84.20

12.70

0.90

1.90

0.40

\section{CONCLUSIONES}

Las relaciones biométricas obtenidas para $A$. trapesialis, están dadas por las siguientes ecuaciones.
(a) Peso total $=0.13172 *$ L ongitud 2,6153
0.873638
(b) Peso parte comestible $=1.05439 \mathrm{EXP}(0.276179 *$ Longitud $) 0.817877$
(c) Peso parte comestible $=-2.47421+0.359364 *$ Peso total 0.811715
(d) Peso parte com. precoc. $=-1.33702+0.20108 *$ Peso total 0.864408

La composición química de la parte comestible fue la siguiente: Agua, $84.20 \%$, proteína $12.60 \%$, grasa, $0.90 \%$, cenizas, $1.90 \%$ y carbohidratos, 0.40 .

A. trapesialis conocida comúnmente como "cuchara', constituye un recurso potencial de la zona de selva, y específicamente de la región San Martín, de buen valor nutricional. 


\section{BIBLIOGRAFIA}

A.O.A.C., 1970. Official Methods of Analysis, Association of Official A griculture Chemists, 1 la. Ed., USA.

CAMPOS, L. 1973. Estudio Brornatológico y de algunos aspectos biológicos de la Anodontites trapesialis del Lago Sauce, Tesis Bachiller Ciencias Biológicas, Trujillo, Perú, U.N. La Libertad, 45 p.

COLLAZOS, C. et al, 1986. Tabla de composición de los alimentos peruanos, Instituto de Nutrición, Lima, Perú, 50.

LLANOS, F. 1978. Estudio de la deshidratación de la macha (Mesodesrna donacium), Tesis Ing. Pesquero, Lima, Perú, UNALM.

CORDOVA, J. 1985. Composición física y química de las cochas de abanico recolectadas en las principales zonas costeras de Pisco, Rey. Lat. Técn. Alim. Pesq., 2: 26-31.

SANCHEZ, J. y LAM , R. 1970. A Igunas características físicas y químicas de las principales especies para consumo humano y sus rendimientos en productos pesqueros en Perú, IM ARPE.

ROBLES, A. y MENDEZ, M. 1989. Moluscos comerciales del Litoral de Tumbes y Piura, Boletín de Lima, No 63, mayo, 47-70. 


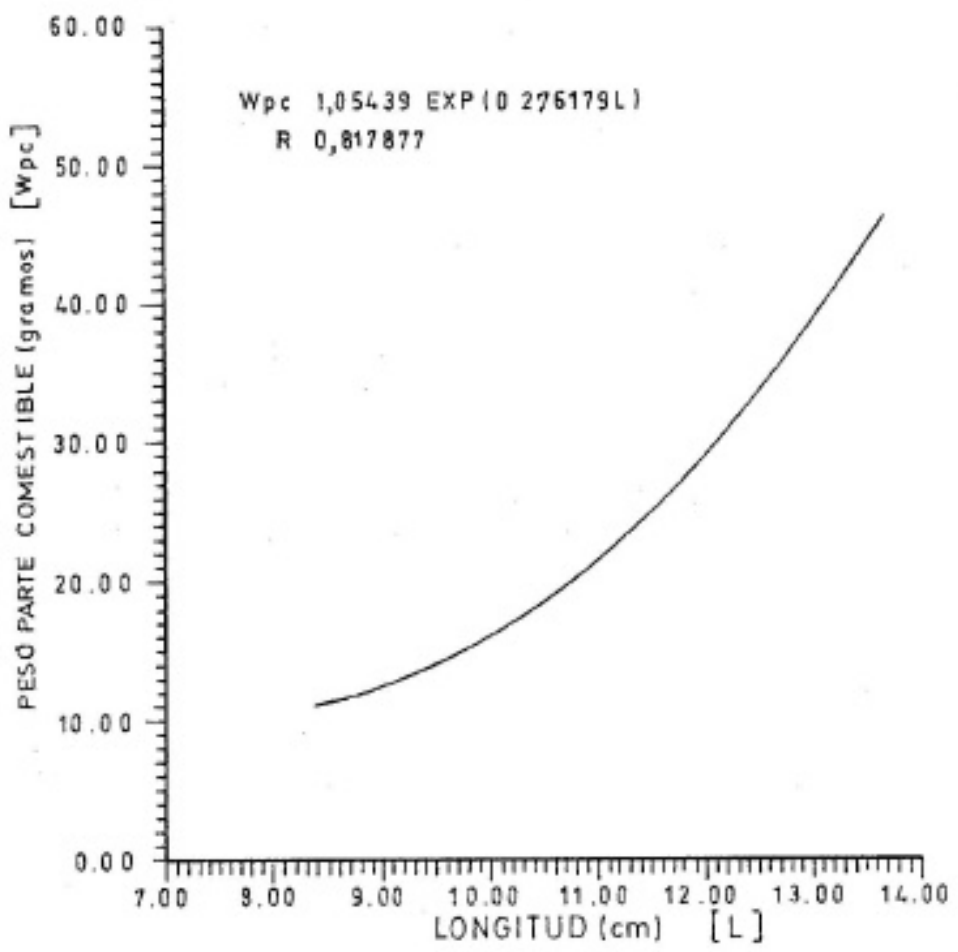

FIG. 3 Relacion peso parte comestible vs longitud de almejas de agua dulce (Anodontites trapesialis) 


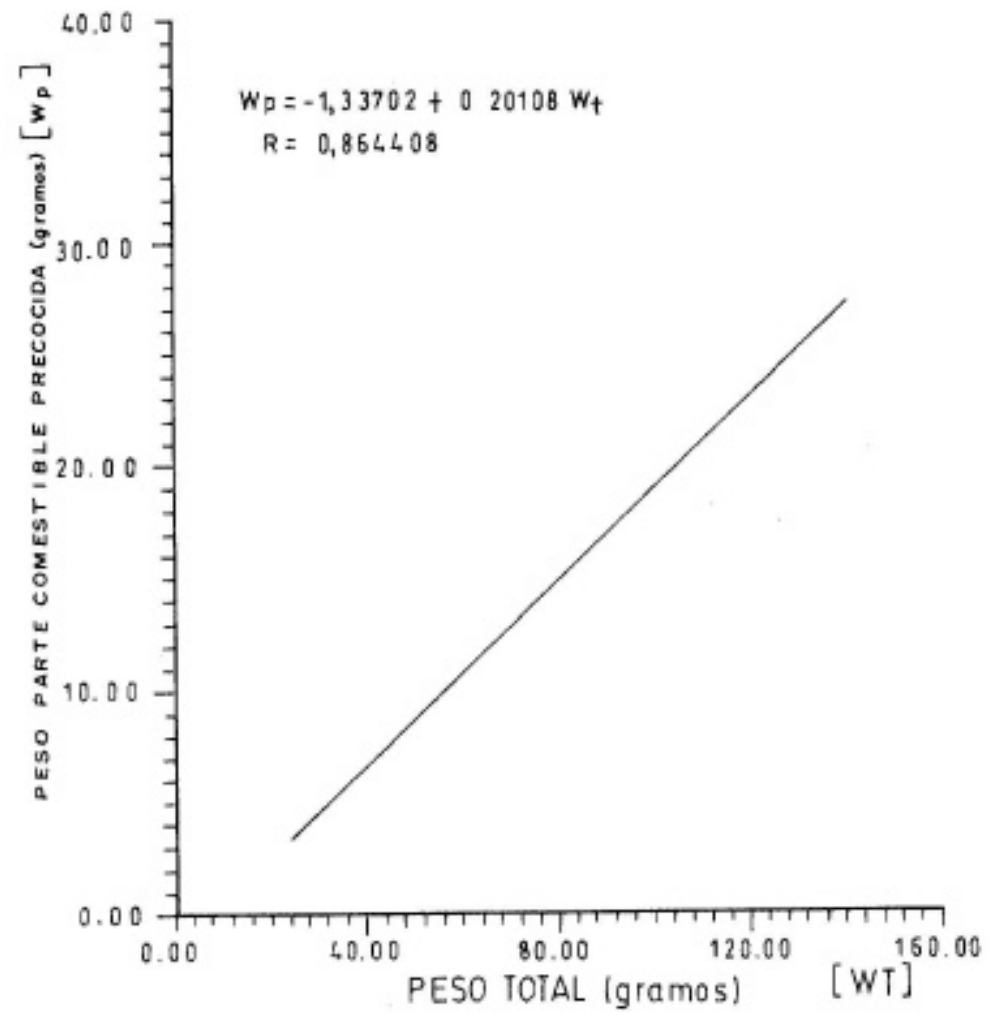

Fig. 5 Relación peso parte comestible precocida vs peso total de almejas de agua dulce (A. trapesiales) 


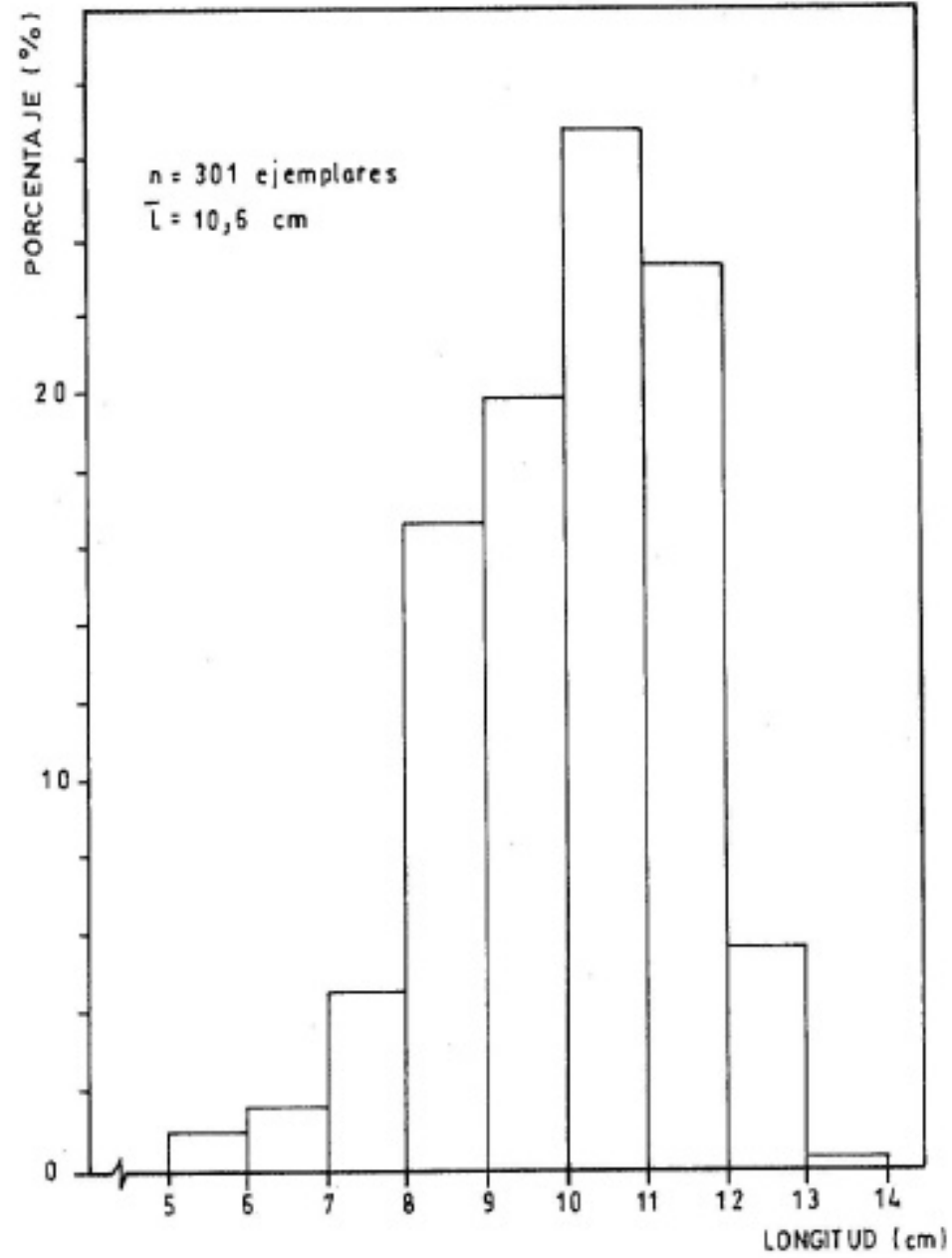

FIG. 6 DISTRIBUCION DE TALLAS DE ALMEJAS DE AGUA DULCE IAnodontitcs trapesialis) 